\title{
PhD theses in constraints
}

\author{
Guido Tack ${ }^{1} \cdot$ Christopher Mears ${ }^{1}$
}

Published online: 20 December 2016

(C) Springer Science+Business Media New York 2016

A significant part of the research in any scientific community is carried out by $\mathrm{PhD}$ students. Due to the nature of a $\mathrm{PhD}$ project, these students often drive innovation and are at the forefront of research in their field. Some of their work is of course published in conference papers and journal articles, but a thesis offers the opportunity to work on a topic in much more depth and breadth than what is typically considered "publishable". The goal of this initiative is to highlight recent work by $\mathrm{PhD}$ students in the area of Constraints. We believe that their work deserves a prominent and easily accessible place in our community. The Association for Constraint Programming has therefore launched a PhD Thesis Archive (http://www.a4cp.org/theses) to collect and promote theses in our field of research. The Constraints journal has kindly agreed to publish a collection of abstracts, and as a service to the community, make them available free of charge. This is the second edition of collected abstracts, for previous years, visit: http://link.springer.com/journal/10601/20/4/page/1.

The topics from these editions span the full spectrum of research in our diverse field, from theoretical to applied, from soft constraints to hard, and with contributions branching out into neighboring fields. The collections include research that has received several best paper and best thesis awards, and has authors from every continent (except Antarctica). If you graduated recently, or plan to submit within the next year, make sure you submit your own abstract, and encourage your colleagues and students to do the same!

Guido Tack and Christopher Mears (guest editors)

Guido Tack

guido.tack@monash.edu

Christopher Mears

chris.mears@monash.edu

1 Monash University, Caulfield East, VIC, 3145, Australia 